$=0$ and $K L^{p}=L$ if and only if every finite-dimensional $L$-module is completely reducible. The latter implies that $\operatorname{Ext}_{V(L)}^{1}(M, N)=0$ for all $V(L)$-modules $M$ and $N$, finite dimensional or not, and therefore all $V(L)$-modules are projective and $V(L)$ has global dimension zero, i.e., $\operatorname{Ext}_{\nabla(L)}^{n}(M, N)=0$ for all $n \geq 1$ and for all $M$ and $N$.

\title{
BIBLIOGRAPHY
} 1-23.

1. S. Eilenberg and J. C. Moore, Limits and spectral sequences, Topology 1 (1961),

2. G. Hochschild, Representations of restricted Lie algebras of characteristic $p$, Proc. Amer. Math. Soc. 5 (1954), 603-605.

3. N. Jacobson, Lie algebras, Interscience, New York, 1962.

4. J. Milnor and J. C. Moore, On the structure of Hopf algebras, Ann. of Math. (to appear).

YALE UNIVERSITY

\section{THE COHOMOLOGY OF THE STEENROD ALGEBRA; STABLE HOMOTOPY GROUPS OF SPHERES ${ }^{1,2}$}

\section{BX J. PETER MAY}

Communicated by W. S. Massey, November 6, 1964

In this paper, we state some of the results obtained by application of the methods of [4] to the study of the cohomology of the Steenrod algebra $A$. In brief, our results are a complete determination of $H^{s, t}(A)$ for $t-s \leqq 42$ in the $\bmod 2$ case, and for $t-s$ $\leqq 2(p-1)\left(2 p^{2}+p+2\right)-4$ in the $\bmod p$ case, $p>2$. Due to the existence of the Adams spectral sequence [1], these results give information about the stable homotopy groups of spheres.

We recall that the $\bmod p$ Adams spectral sequence $\left\{E_{r}\right\}$ (for the sphere) has differentials $\delta_{r}: E_{r}^{s, t} \rightarrow E_{r}^{s+r, t+r-1}$ and satisfies the properties:

$$
E_{2}^{s, t} \cong H^{s, t}(A) \text { as a } Z_{p} \text {-algebra. }
$$

(3) $\left\{E_{\infty}^{s, t} \mid t-s=k\right\}$ provides a composition series for $\pi_{k}(S ; p)$ (relative to a suitable filtration); here $\pi_{k}(S, p)$ denotes the stable homotopy group $\pi_{k}(S)$ modulo the subgroup of elements whose order is finite and prime to $p$.

${ }^{1}$ During the preparation of this paper, the author was partially supported by National Science Foundation grant number NSF-GP-1853.

2 The work announced here is contained in the author's doctoral thesis, submitted to Princeton University. 
Now Toda [5], [6] has calculated $\pi_{k}(S, 2), k \leqq 19$, and $\pi_{k}(S, p), p>2$, $k \leqq 2 p^{2}(p-1)-3$, with partial information for $k=2 p^{2}(p-1)-2$ and $2 p^{2}(p-1)-1$. It turns out that there is only one pattern of differentials consistent with Toda's results. Therefore the structure of the Adams spectral sequence is completely known in the range of Toda's computations. Partial information about the differentials in higher dimensions can be obtained whenever an element is decomposable in terms of elements whose differentials are known. In fact, there are enough relations to allow complete determination of $\pi_{k}(S, 2), k \leqq 28$, and to determine $\pi_{k}(S, p), p>2, k<2(p-1)\left(p^{2}+2 p\right)-4$, up to one unknown differential.

We state our results first for the case $p=2$. Let $H^{k}(A)$ $=\otimes_{t-s=k} H^{s, t}(A) . H^{k}(A)$ is a $Z_{2}$-space, but there exists an element $h_{0} \in H^{1,1}(A)$ such that multiplication by $h_{0}$ on elements surviving to $E_{\infty}$ corresponds essentially to multiplication by 2 in the ring $\pi(S, 2)$. We interpret multiplication by $h_{0}$ in $H^{*}(A)$ to be multiplication by 2 in writing the groups $H^{k}(A)$ below. We only state our results in those dimensions in which nearly complete information on $\pi_{k}(S, 2)$ has been obtained.

TheOREM 1. For $18 \leqq k \leqq 31, H^{k}(A)$ and $\pi_{k}(S, 2)$ are as follows:

\begin{tabular}{lll}
$k$ & \multicolumn{1}{c}{$H^{k}(A)$} & $\pi_{k}(S, 2)$ \\
18 & $Z_{2}+Z_{4}+Z_{8}$ & $Z_{2}+Z_{8}$ \\
19 & $Z_{2}+Z_{8}$ & $Z_{2}+Z_{8}$ \\
20 & $Z_{8}$ & $Z_{8}$ \\
21 & $Z_{2}+Z_{2}$ & $Z_{2}+Z_{2}$ \\
22 & $Z_{8}+Z_{2}$ & $Z_{2}+Z_{2}$ \\
23 & $Z_{2}+Z_{4}+Z_{8}+Z_{16}$ & $Z_{2}+Z_{2}+Z_{8}+Z_{16}$ \\
24 & $Z_{2}+Z_{2}+Z_{2}$ & $Z_{2}+Z_{2}$ \\
25 & $Z_{2}+Z_{2}+Z_{16}$ & $Z_{2}+Z_{2}$ \\
26 & $Z_{2}+Z_{2}+Z_{8}$ & $Z_{2}+Z_{2}$ \\
27 & $Z_{8}$ & $Z_{8}$ \\
28 & $Z_{8}$ & $Z_{2}$ \\
29 & $Z_{8}$ & 0 or $Z_{2}$ \\
30 & $Z_{2}+Z_{8}+Z_{16}+Z_{32}$ & $0 \subseteq \pi_{30}(S, 2) \subseteq\left(Z_{2}\right)^{3}$ \\
31 & $\left(Z_{2}\right)^{3}+Z_{8}+Z_{216}$ & $Z_{64} \subseteq \pi_{31}(S, 2) \subseteq Z_{64}+\left(Z_{2}\right)^{4}$
\end{tabular}

(here $\left(Z_{2}\right)^{n}=Z_{2}+\cdots+Z_{2}, n$ summands). 
REMarks. Adams [2] has calculated $H^{*, t}(A)$ for $t-s \leqq 17$ and for $t \leqq 24$. Liulevicius (unpublished) has calculated $H^{s, t}(A)$ for $t \leqq 31$, which, together with the periodicity theorems of Adams [2], gives complete information for $t-s \leqq 23$. The stated facts about $\pi_{k}(S, 2)$, $k=30$ and $k=31$, follow from unpublished results of Maunder, in which he identifies the elements of $E_{2}$ which survive to the image of the $J$-homomorphism $Z_{2^{n+1}} \subset \pi_{k}(S, 2), k=2^{n}+2^{n+1} m-1, m \geqq 0, n \geqq 2$.

Next we state our results for the case $p>2$. As in the case $p=2$, there is an element $a_{0} \in H^{1,1}(A)$ such that multiplication by $a_{0}$ on elements surviving to $E_{\infty}$ corresponds essentially to multiplication by $p$ in $\pi(S, p)$, and we may define a group structure on each $E_{r}^{\boldsymbol{k}}=\otimes_{t-s=k} E_{r}^{s, t}$ by interpreting multiplication by $a_{0}$ to mean multiplication by $p$. For the sake of brevity, we state our results in terms of $E_{4}$ and restrict our attention to those dimensions $k$ in which nearly complete information on $\pi_{k}(S, p)$ is obtained.

THEOREM 2. The following elements (of the stated orders and dimensions) generate all $E_{4}^{k}$ in the range $0<k \leqq 2(p-1)\left(p^{2}+2 p\right)-4$ : (In the list below, $j \geqq 1$ and $q=2(p-1))$.

\begin{tabular}{lcl} 
element & order & \multicolumn{1}{c}{ dimension } \\
$\alpha_{j}$ & $p$ & $j q-1, j \neq 0 \bmod p$ \\
$\alpha_{j p}$ & $p^{2}$ & $j p q-1, j \neq 0 \bmod p$ \\
$\alpha_{j p^{2}}^{\prime \prime}$ & $p^{3}$ & $j p^{2} q-1, j \neq 0 \bmod p$ \\
$\beta_{l+1} \beta_{1}^{j-1}$ & $p$ & $(j p+l p+l) q-2 j, 0 \leqq l \leqq p-2$ \\
$\alpha_{1} \beta_{l+1} \beta_{1}^{j-1}$ & $p$ & $(j p+l p+l+1) q-2 j-1,0 \leqq l \leqq p-2$ \\
$\beta_{l+1} \beta_{1}^{j-1} \gamma$ & $p$ & $\left(p^{2}+j p+l p+l\right) q-2 j-2,0 \leqq l \leqq p-2$, \\
& & $\left(p^{2}+j p+l p+l+1\right) q-2 j-3, \quad 0 \leqq l \leqq p-2$, \\
$\alpha_{1} \beta_{l+1} \beta_{1}^{j-1} \gamma$ & $p$ & \\
& $p$ & $\left(p^{2}+l\right) q-3,1 \leqq l \leqq p-2$ \\
$\alpha_{l} \gamma$ & $p$ & $\left(p^{2}+l\right) q-2,1 \leqq l \leqq p-1$ \\
$\epsilon_{l}$ & $p^{2}$ & $\left(p^{2}+p\right) q-3$ \\
$\phi$ & $p$ & $\left(p^{2}+j p+p-1\right) q-2 j-2, j=1$ if $p=3$.
\end{tabular}

$\delta_{2 p-1}\left(\beta_{l+1} \beta_{1}^{j-1} \gamma\right)=\alpha_{1} \beta_{l+1} \beta_{1}^{p+j-1}$ or zero. All listed elements other than the $\beta_{l+1} \beta_{1}^{j-1} \gamma$ are cocycles under each $\delta_{r}$, and, at least in the range $0<k$ $<2(p-1)\left(p^{2}+2 p\right)-4$, no listed elements other than the $\alpha_{1} \beta_{l+1} \beta_{1}^{p+g-1}$ are coboundaries under any $\delta_{r}$. 
REMARKs. In [5, III, p. 209], Toda states a problem equivalent to the problem of determining $\delta_{2 p-1}(\gamma)$. Gershenson [3] has calculated $H^{s, t}(A)$ for $t-s \leqq 2 p(p-1)-1$. The results due to Milnor and Kervaire on the image of the $J$-homomorphism imply that for each $n \geqq 0$ and $j \geqq 1, j \neq 0 \bmod p$, there will be an element $\alpha_{j p^{n}}^{(n+1)}$, of order $p^{n+1}$ in $\pi_{k}(S, p), k=2 j p^{n}(p-1)-1$.

Proofs and a complete statement of our results will appear elsewhere.

\section{BIBLIOGRAPHY}

1. J. F. Adams, On the structure and applications of the Steenrod algebra, Comm. Math. Helv. 32 (1958), 180-214.

2. - Stable homotopy theory (lecture notes), Univ. of California, Berkeley, Calif., 1961.

3. H. H. Gershenson, Relationships between the Adams spectral sequence and Toda's calculations of the stable homotopy groups of spheres, Math. Z. 81 (1963), 223259.

4. J. P. May, The cohomology of restricted Lie algebras and of Hopf algebras, Bull. Amer. Math. Soc. 71 (1965), 372-377.

5. H. Toda, p-primary components of homotopy groups. I, Exact sequences in the Steenrod algebra. II, mod p Hopf invariant. III, Stable groups of the sphere. IV, Compositions and toric constructions, Mem. College Sci. Univ. Kyoto 31 (1958), 129-142; 143-160; 191-210; ibid. 32 (1959), 297-332.

6. - Composition methods in homotopy groups of spheres, Princeton Univ. Press, Princeton, N. J., 1962.

YALE UNIVERSITY 